\title{
ESTUDO DO FENÔMENO DE FRATURA INTERGRANULAR ASSISTIDO POR OXIDAÇÃO NA INCONEL 718*
}

\section{Resumo}

\author{
Amanda de Vasconcelos Varela ${ }^{1}$ \\ Henrique Dias de Deus ${ }^{2}$ \\ Monica Costa Rezende ${ }^{3}$ \\ Luiz Henrique de Almeida ${ }^{4}$
}

A Inconel 718 tem sido usada por décadas nas indústrias aeroespacial e nuclear por sua boa combinação de resistência mecânica e resistência a oxidação. No entanto, entre aproximadamente 650 e $850^{\circ} \mathrm{C}$, observa-se uma mudança indesejável no modo de fratura da superliga, que caracteriza a fratura intergranular assistida por oxidação. Com as temperaturas de operação em cada vez maiores e uma alta demanda por eficiência energética, a fragilização pelo ambiente é uma grande limitação a aplicação da superliga. Por isso, o objetivo deste trabalho é caracterizar o fenômeno através de ensaios de tração a quente em diferentes taxas de deformação e por microscopia eletrônica de varredura e EDS. A faixa de temperatura de ocorrência do fenômeno, segundo a evidência de fratura frágil intergranular variou conforme a taxa aplicada, manifestando-se entre 650 e $750^{\circ} \mathrm{C}$ até $900^{\circ} \mathrm{C}$ em todos os casos. O efeito da disponibilidade de oxigênio foi observado e o elemento não demonstrou ser determinante na perda de propriedades mecânicas avaliadas em tração. O nióbio, presente em quantidade considerável na liga, apareceu predominantemente como $\mathrm{NbC}$ nos contornos e interior dos grãos, incorporado ao titânio na região de fratura, com aspecto fragmentado. Acredita-se, portanto, que este tenha papel decisivo no processo de fragilização.

Palavras-chave: Inconel 718; Oxidação; Fratura; Nióbio.

\section{STUDY OF THE OXIDATION ASSISTED INTERGRANULAR CRACKING ON INCONEL 718}

\section{Abstract}

Inconel 718 has been used for decades in nuclear and aerospace industries due to its good combination of strength and oxidation resistance. However, approximately between 650 and $850^{\circ} \mathrm{C}$, a transition in the fracture mode is observed, revealing an intergranular fracture. With increasing operation temperatures and efficiency requirements, environmental embrittlement has become a relevant source of limitation to this alloy's applications. Because of this, OAIC has been characterized by hot tensile testing at different deformation rates and scanning electron microscopies plus EDS. The phenomenon had different onset temperatures for each deformation rate, suggested by brittle fracture evidence on the fractured surfaces, starting between 650 and $750^{\circ} \mathrm{C}$, while it was finished at $900^{\circ} \mathrm{C}$ for all conditions. Oxygen availability was considered and it was not shown to be crucial to the loss of mechanical properties. Oppositely, niobium, which is highly present at this alloy, was identified as $\mathrm{NbC}$ with incorporated titanium precipitated along and within grain boundaries in the fracture region. Therefore, it might a play major role on OAIC.

Keywords: 718 Alloy; Oxidation; Fracture; Niobium.

1 Engenheira de Materiais, BS, Mestranda, Programa de Engenharia Metalúrgica e de Materiais, UFRJ, Rio de Janeiro, RJ, Brasil.

2 Estudante de Graduação, Programa de Engenharia Metalúrgica e de Materiais, UFRJ, Rio de Janeiro, RJ, Brasil.

3 Engenheira Metalúrgica, Doutora, Pós-Doutoranda, Programa de Engenharia Metalúrgica e de Materiais, UFRJ, Rio de Janeiro, Brasil

4 Engenheiro Metalúrgico, Doutor, Professor, Programa de Engenharia Metalúrgica e de Materiais, UFRJ, Rio de Janeiro, Brasil. 


\section{INTRODUÇÃO}

As superligas de níquel representam uma classe de materiais desenvolvida com objetivo de suportar longos tempos de serviço em ambientes extremos, como exposição a altas temperaturas e meios corrosivos, mantendo elevado desempenho. A Inconel 718 é a superliga mais utilizada e produzida atualmente, representando cerca de $50 \%$ da produção de superligas comerciais [1]. Esta liga a base de níquel, ferro e cromo destaca-se por sua combinação de propriedades, que inclui alta resistência e boas conformabilidade e usinabilidade. Descrita como complexa, esta liga possui grande variedade de elementos químicos, com principais adições de alumínio, titânio, nióbio e molibdênio.

A utilização destas superligas em aplicações industriais é extremamente dependente de sua integridade em serviço em altas temperaturas. Por isso, é necessária atenção a possíveis variações na microestrutura, que podem comprometer este bom desempenho. A literatura relata que em exposição ao ar, entre 500 e $800^{\circ} \mathrm{C}$, é possível observar perda de ductilidade e fratura intergranular, comumente associada ao mecanismo conhecido por OAIC (Oxidation Assisted Intergranular Cracking). Este fenômeno sobrepõe o efeito protetor da barreira formada pelos óxidos de cromo e alumínio, uma vez que o oxigênio do ambiente difunde-se rapidamente e de maneira preferencial pelos contornos de grão, fragilizando o material e o levando a fraturar prematuramente [2].

Alguns trabalhos que investigaram o OAIC em superligas de níquel deformadas a temperaturas elevadas, embora reconheçam a influência do oxigênio no modo de fratura, demonstram que não existe um consenso em relação às temperaturas de início e fim do fenômeno, assim como a explicação metalúrgica para seu acontecimento. O efeito do ambiente, inclusive, é apenas um componente necessário ao processo deletério, mas não suficiente para que ele ocorra [3]. Por isso, a interpretação dada ao crescimento de trincas nestes materiais ainda é controversa. Dessa forma, o presente trabalho tem como objetivo estudar os mecanismos do OAIC em superligas 718. Para tanto, foi feito ensaio de tração a quente e posterior análise de imagem por microscopia eletrônica de varredura com EDS para estudar as superfícies de fratura.

\subsection{Revisão da Literatura}

\subsubsection{Inconel 718}

A Inconel 718 é uma liga de matriz níquel-ferro-cromo presente em diferentes indústrias como as nucleares, aeroespaciais e de óleo e gás. Além do bom desempenho em solicitações de altas pressões/tensões e ambientes corrosivos, a superliga tem relativo baixo custo pela substituição de alguns elementos como alumínio, cobalto, titânio e tungstênio por ferro e nióbio. Quanto à microestrutura, essas ligas de matriz austenítica $y$ são formadas por uma grande fração volumétrica de $\gamma^{\prime}$ e $\gamma^{\prime \prime}$, eventual presença da fase $\delta$ e carbetos majoritariamente do tipo MC.

A matriz y é, então, cfc e estável em todo intervalo de temperaturas de aplicação. A fase Y' ((Al,Ti) $\left.)_{3} \mathrm{Ni}\right)$ cfc é endurecedora e estável em altas temperaturas pois, apesar da rápida precipitação, sua taxa de crescimento é lenta. É formada principalmente por alumínio ou titânio. Já a fase $\mathrm{Y}$ " $\left(\mathrm{Ni}_{3} \mathrm{Nb}\right)$ tetragonal é a principal fase endurecedora desta liga, metaestável, formada pelo nióbio. Possui baixíssima cinética de precipitação, o que confere melhor conformabilidade e soldabilidade, 
além da elevada cinética de crescimento acima de $800^{\circ} \mathrm{C}$, quando o precipitado evolui para a fase $\delta$. Esta última tem a mesma fórmula estequiométrica da anterior, porém apresenta estrutura cristalina ortorrômbica. Pode ser precipitada em baixas ou altas temperaturas, em torno de $750^{\circ} \mathrm{C}$ ou entre 900 e $1000^{\circ} \mathrm{C}$, durante $\mathrm{o}$ tratamento de solubilização. Por fim, os carbetos MC são comumente encontrados nas formas de $\mathrm{NbC}$ e TiC. Esses carbetos de estrutura cúbida formam-se durante a solidificação.

O grande diferencial da IN-718 em relação a outras ligas a base de níquel é seu elevado teor de nióbio. Este elemento refratário de alto ponto de fusão forma a principal fase endurecedora $\mathrm{Y}^{\prime}$, como mencionado, a qual é geralmente associada as boas propriedades mecânicas da liga. $\mathrm{O}$ nióbio ainda aumenta a quantidade da fase ordenada $\mathrm{Y}^{\prime}$, pois pode substituir parcialmente o alumínio nesta fase, além de favorecer a formação de MC. Por este carbeto estável ser rico em nióbio e precipitar principalmente nos contornos de grão, pode ter influência significativa no modo de fratura da liga, como destacado em [4]. Uma grande concentração de precipitados nos contornos, resultado de tempo prolongado de envelhecimento ou elevada temperatura de tratamento, gera uma mistura de fratura transgranular e integranular, até que seja predominantemente integranular, com a formação de uma camada quase contínua de carbetos.

\subsubsection{Tratamentos térmicos}

Os tratamentos térmicos são parte do processamento da superliga e tem como objetivo modificar a estrutura do material pelo aumento de temperatura. Para essa liga, os tratamentos comumente aplicados são a solubilização e envelhecimento, com uma ou mais etapas de precipitação. Ao fim, o controle do tamanho e distribuição dos precipitados adequará as propriedades definidas para as aplicações específicas de cada tipo de indústria.

A solubilização é a etapa que consiste em homogeneizar a microestrutura, eliminando quaisquer segundas fases, com o objetivo de preparar o material para o envelhecimento, embora a dissolução de algumas fases possa não ocorrer. $\mathrm{Na}$ literatura, é possível encontrar temperaturas de solubilização entre 955 e 1200ㄷ․ A escolha desta temperatura é especialmente relevante na produção de $\delta$, solubilizado entre 970 e $1050^{\circ} \mathrm{C}$. Após essa etapa, a liga pode ser resfriada ao ar, em água ou em óleo, por exemplo.

O envelhecimento na Inconel 718 é normalmente realizado em duas etapas para gerar a precipitação das fases endurecedoras $\gamma^{\prime}$ e $y^{\prime \prime}$, responsáveis pela resistência mecânica da liga, e controlar a morfologia dos carbetos no contorno de grão. As fases Y' e Y" precipitam rapidamente entre 700 e $900^{\circ}$. Mantendo esta temperatura inferior a $800^{\circ} \mathrm{C}$, além do controle do tamanho de grão, impede-se a formação de $\delta$.

\subsubsection{Microestrutura}

Durante os tratamentos térmicos da liga, há a precipitação tanto das fases metaestáveis $Y^{\prime}$ e $Y$ ", quanto de carbetos primários e secundários. O carbeto predominante observado na Inconel 718 é do tipo MC, especialmente rico em nióbio. A microestrutura da IN-718 solubilizada em temperatura suficientemente alta para promover a dissolução das fases irá apresentar uma matriz CFC y e carbetos MC, 
em sua maioria de nióbio e também de titânio, com microestrutura típica apresentada a seguir pela Figura 1. A microestrutura envelhecida, por sua vez, vai apresentar a precipitação das fases endurecedoras e maior fração de $\mathrm{Y}$ " com a temperatura. Ela é exemplificada também na Figura 1.
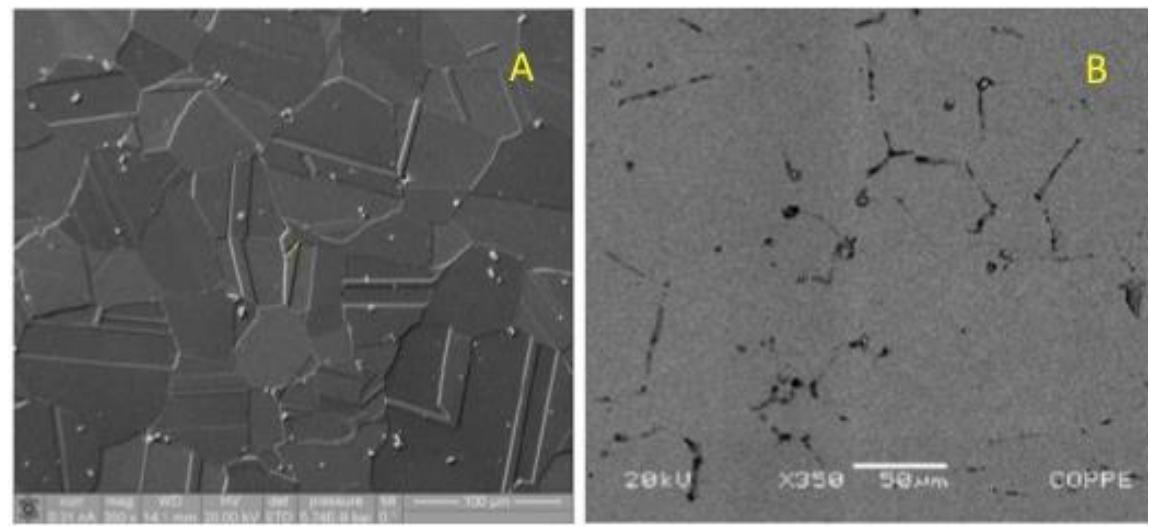

Figura 1 - Microestrutura (MEV) típica da Inconel 718 solubilizada (A) e envelhecida (B). Na primeira os grãos aproximadamente equiaxiais apresentam muitas maclas e os carbetos $\mathrm{MC}$ distribuídos. $\mathrm{Na}$ segunda, a precipitação das fases endurecedoras é distinguível apenas nos contornos com a presença de $\mathrm{Y}$ ".

\subsubsection{Propriedades Mecânicas}

Pela Figura 2 pode-se observar a evolução a curto prazo das principais propriedades mecânicas até $1000^{\circ} \mathrm{C}$ da liga Inconel 718 solubilizada e envelhecida. Pode-se observar uma piora das propriedades mecânicas a partir de cerca de $650^{\circ} \mathrm{C}$, indicando a existência de mecanismos degradativos a partir dessa temperatura.

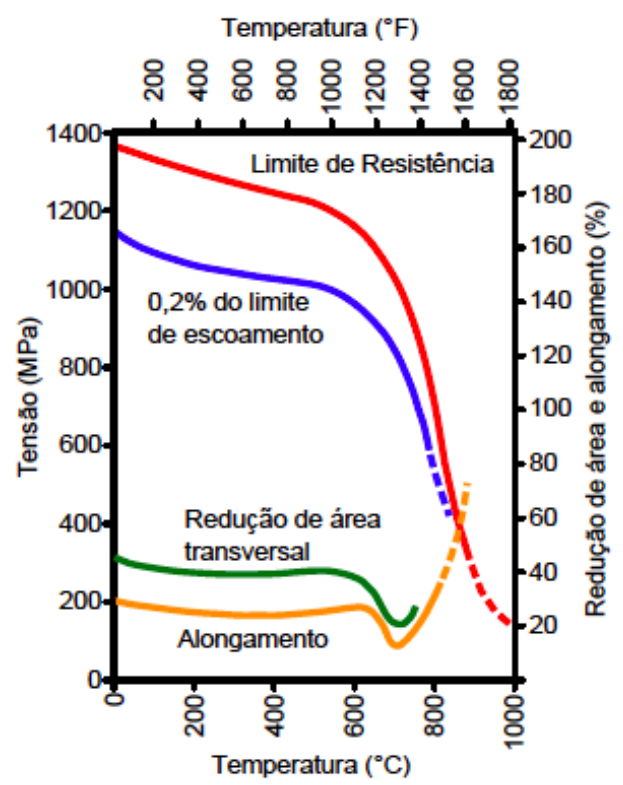

Figura 2 - Propriedades mecânicas da Inconel 718 envelhecida. Em torno de $650^{\circ} \mathrm{C}$ observa-se um decréscimo das propriedades avaliadas [5]. 


\subsubsection{Fragilização pelo ambiente (OAIC)}

Como comentado anteriormente, as condições nas quais a Inconel 718 é aplicada requerem principalmente boa resistência mecânica e resistência a oxidação a temperaturas elevadas. Estudos das décadas de 70 e 80 abordaram amplamente a penetração de oxigênio nas superligas, que aumenta com 0 tempo e a temperatura, tornando-as suscetíveis a fragilização. No entanto, a degradação das propriedades observadas era máxima em temperaturas intermediárias [6]. Por isso, as superligas de níquel tem sido estudadas extensivamente com objetivo de analisar os fenômenos que afetam sua microestrutura e propriedades.

O motivo para que essa liga possua boas propriedades contra a oxidação é a presença, em sua composição, de ao menos um elemento que se oxide e produza um óxido protetor. Para que isso aconteça, a camada de óxido deve ser contínua sobre a superfície, além de possuir nela baixa taxa de difusão das espécies reativas. O conceito, chamado oxidação seletiva, é observado nas superligas de níquel através do alumínio e/ou cromo, que são responsáveis pela formação dos óxidos estáveis $\mathrm{Al}_{2} \mathrm{O}_{3}$ e $\mathrm{Cr}_{2} \mathrm{O}_{3}$, respectivamente. As fases que não contém estes elementos são preferencialmente oxidadas, como os carbetos.

A oxidação que leva à fragilização da Inconel 718 é observada em um intervalo de temperaturas intermediário, no qual a liga passa por uma transição no modo de fratura. Esta deixa de ser dúctil e se torna intergranular, como resultado do enfraquecimento dos contornos de grão. Isso caracteriza o fenômeno conhecido como OAIC. Por isso, a garantia de estabilidade estrutural do material é normalmente limitada a $650^{\circ} \mathrm{C}$. No entanto, a temperatura de trabalho de um disco de turbina feito da superliga é entre 426 e $760^{\circ} \mathrm{C}$, por exemplo, o que torna o tema de grande relevância industrial. Além disso, a explicação metalúrgica para 0 mecanismo é pouco esclarecida em cerca de 30 anos de pesquisa.

Dessa forma, algumas hipóteses foram tratados por diversos autores. Há a premissa de que o fenômeno de oxidação tem papel dominante do oxigênio. Porém, pode-se destacar que o oxigênio não age como fragilizante em sua forma elementar. $\mathrm{Na}$ Inconel 718 especificamente, o nióbio presente em quantidade considerável na liga é caracterizado por sua afinidade ao oxigênio, que tem solubilidade crescente na matriz de nióbio com a temperatura. Por isso, a maioria dos estudos devem a este elemento de liga o papel crucial no mecanismo de oxidação em altas temperaturas. Ainda, a segregação preferencial do nióbio nos contornos indica que o elemento deva desempenhar papel importante no crescimento de trincas influenciadas pelo ambiente em altas temperaturas. Inicialmente, o papel do $\mathrm{NbC}$ foi discutido e apontado como precursor da formação do óxido frágil $\mathrm{Nb}_{2} \mathrm{O}_{5}$, produto de sua reação com o oxigênio. A principal fonte de nióbio que contribui para o fenômeno, no entanto, não é esclarecida [7,8].

Por fim, em estudos de temperaturas mais elevadas, acima de $850^{\circ} \mathrm{C}$, é possível observar a recuperação da ductilidade do material e da resistência a oxidação característica das superligas de níquel [9]. Esta é atribuída à formação de $\mathrm{Cr}_{2} \mathrm{O}_{3}$, que passa a ser termodinamicamente mais favorável e cresce preferencialmente sobre os contornos. Como consequência, a taxa de oxidação decresce rapidamente pois dificulta a difusão de algumas espécies químicas sobre ela, como o nióbio. 


\section{MATERIAIS E MÉTODOS}

O material utilizado foi fabricado e fornecido pela Villares Metals. A composição química da liga, fornecida pela Villares Metals, é apresentada na Tabela 1.

Tabela 1. Composição química (\% em peso) da Inconel 718

\begin{tabular}{lccccccccccc}
\hline $\mathbf{N i}$ & $\mathbf{C r}$ & $\mathbf{T i}$ & $\mathbf{C o}$ & $\mathbf{M o}$ & $\mathbf{C u}$ & $\mathbf{N b}$ & $\mathbf{M n}$ & $\mathbf{A l}$ & $\mathbf{F e}$ & $\mathbf{C}$ & $\mathbf{P ~ e ~ S}$ \\
\hline 52,3 & 18,4 & 1,02 & 0,042 & 2,8 & 0,026 & 5,1 & 0,017 & 0,57 & 18,2 & 0,04 & $<0,005$ \\
\hline
\end{tabular}

A liga Inconel 718 foi recebida em forma de eletrodos, oriundos de lingotes fundidos em dupla etapa, que foram refundidos no VAR instalado no Laboratório Multiusuário de Fusão a Arco da COPPE/UFRJ. O processamento incluiu a homogeneização, forjamento e laminação a quente dos lingotes, gerando o produto final, que constituiu de barras redondas com diâmetro de $41 \mathrm{~mm}$. As barras foram posteriormente cortadas pelo processo de eletroerosão, na empresa HR Mecânica. Por fim, a usinagem do material foi realizada pela Usifer, gerando os corpos de prova de tração de seção transversal circular com as dimensões estabelecidas pela norma EN 10002-5.

Um tratamento térmico foi conduzido no material com objetivo de produzir amostras na condição solubilizada. O tratamento foi feito no forno Carbolite CWF 13/23 do laboratório TERMIC da COPPE/UFRJ. Antes serem submetidos ao tratamento, os materiais foram encapsulados em quartzo a vácuo, como medida de prevenção a oxidação superficial das amostras. A solubilização foi realizada a $1050^{\circ} \mathrm{C}$ durante 1 hora, para a completa dissolução das fases. A temperatura, embora seja superior as industriais, é interessante para produzir uma liga livre de precipitados $\delta$. O material foi resfriado em água.

Os ensaios de tração foram conduzidos a quente, sob vácuo de $1,5 \times 10^{-2} \mathrm{mbar}$, na máquina de tração universal da marca EMIC, modelo DL 10000, do laboratório de Propriedades Mecânicas da COPPE/UFRJ. A máquina continha um forno tubular acoplado, da marca Instron. A temperatura de teste foi monitorada por um termopar próximo ao corpo de prova, fixado no objeto lançador que faz a inserção da amostra no interior do forno. O intervalo de temperaturas dos ensaios compreendeu de 600 a $925^{\circ} \mathrm{C}$ e as taxas de deformação impostas foram três, referidas como baixa, intermediária e alta, correspondentes a $3,2 \times 10^{-5}, 3,2 \times 10^{-4}$ e $3,2 \times 10^{-3} \mathrm{~s}^{-1}$, respectivamente.

Para uso da técnica de microscopia eletrônica de varredura de alta resolução (MEV-FEG), foi utilizado o equipamento VERSA, instalado no Núcleo de Microscopia Eletrônica da COPPE/UFRJ. Esta etapa teve como objetivos principais a geração de perfis de composição na região dos contornos, onde ocorre o OAIC, e realizar o mapeamento da região do contorno de amostras que evidenciassem o início e o fim do fenômeno. Os constituintes da microestrutura foram identificados pelo sistema Energy Dispersive Spectroscopy (EDS).

\section{RESULTADOS E DISCUSSÃO}

A Figura 2 apresenta os principais resultados dos ensaios de tração a quente, representados pela taxa intermediária. $O$ aumento da temperatura causou queda no limite de resistência e ductilidade dentro de um intervalo de temperaturas para as 
três taxas. Para todos os casos, a ductilidade foi parcialmente recuperada a partir de $900^{\circ} \mathrm{C}$. A avaliação dos parâmetros de resistência, pelo limite de escoamento (LE), e ductilidade, pela elongação, para a taxa intermediária é representada pelo gráfico da Figura 3 e ilustra melhor este comportamento.

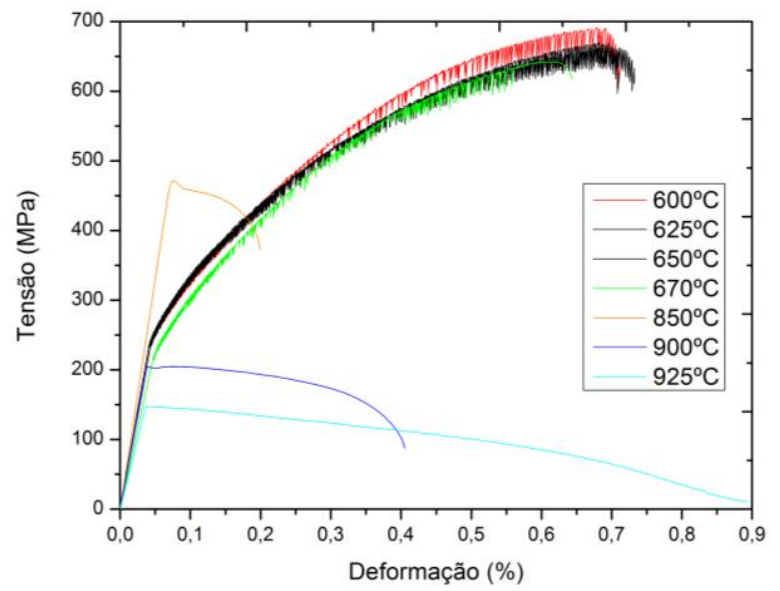

Figura 2 - Curvas de tração das amostras ensaidas entre 600 e $925^{\circ} \mathrm{C}$, indicando a perda nas propriedades mecânicas a partir de $670^{\circ} \mathrm{C}$.

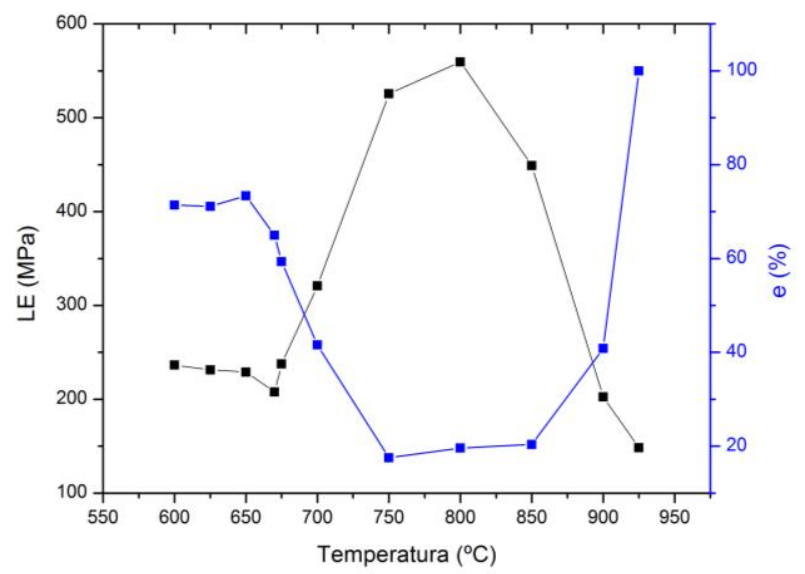

Figura 3 - Avaliação das propriedades em tração. A região de maior queda de ductilidade corresponde ao OAIC.

Embora o aumento no LE seja esperado para o intervalo com a presença do envelhecimento dinâmico e da precipitação de $\gamma^{\prime}$ e $\gamma^{\prime \prime}$, a acentuada queda na elongação indica a presença de outro fenômeno, o de oxidação. Assim, as superfícies de fratura foram analisadas no MEV para confirmar a transição do modo de fratura (Figura 4). O ensaio a $600^{\circ} \mathrm{C}$ mostrou superfície de dimples, típica da fratura dúctil. Já a $670^{\circ} \mathrm{C}$ foi possível observar evidência de fratura intergranular apenas nas bordas do corpo de prova. À $850^{\circ} \mathrm{C}$, na região do mínimo de ductilidade, a fratura foi $100 \%$ intergranular. $\mathrm{E}$, por fim, a $900^{\circ} \mathrm{C}$ o material que mostrou recuperação nas propriedades apresentou também aspecto mais dúctil. Na taxa mais rápida a temperatura de início da fratura frágil foi $750^{\circ} \mathrm{C}$ e na mais lenta, $600^{\circ} \mathrm{C}$.

A fim de identicar o nióbio nas regiões de fratura, também foi realizado mapeamento por EDS no microscópio de alta resolução. Como apresentado na Figura 5, o elemento apareceu marcadamente nessas regiões, sempre associado ao titânio e em diferentes aspectos para as temperaturas fora e dentro do intervalo do OAIC. Fora do intervalo foi constatada a presença de carbetos MC alinhados na 
direção de processamento, aproximadamente esféricos e predominantemente ao longo dos contornos de grão. Já com a manifestação do fenômeno, os mesmos aparecem aglomerados nas bordas da fratura, com aspecto fragmentado, aparentemente formando caminhos preferenciais ao longo de contornos e também pelo interior dos grãos. Essa observação é representada pelas temperaturas de ensaio de 600 e $850^{\circ} \mathrm{C}$ na Figura 5.
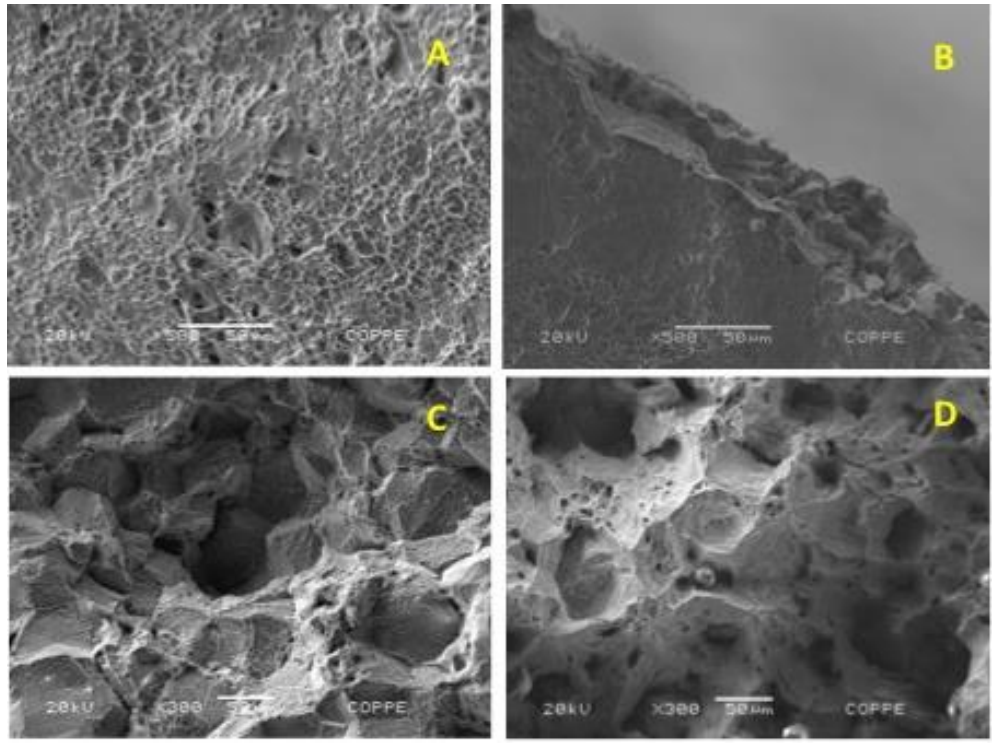

Figura 4 - Micrografias (MEV) da transição no modo de fratura causado pelo OAIC na Inconel 718, a partir dos ensaios a (a) $600^{\circ} \mathrm{C}$, (b) $670^{\circ} \mathrm{C}$, (c) $850^{\circ} \mathrm{C}$ e (d) $900^{\circ} \mathrm{C}$.
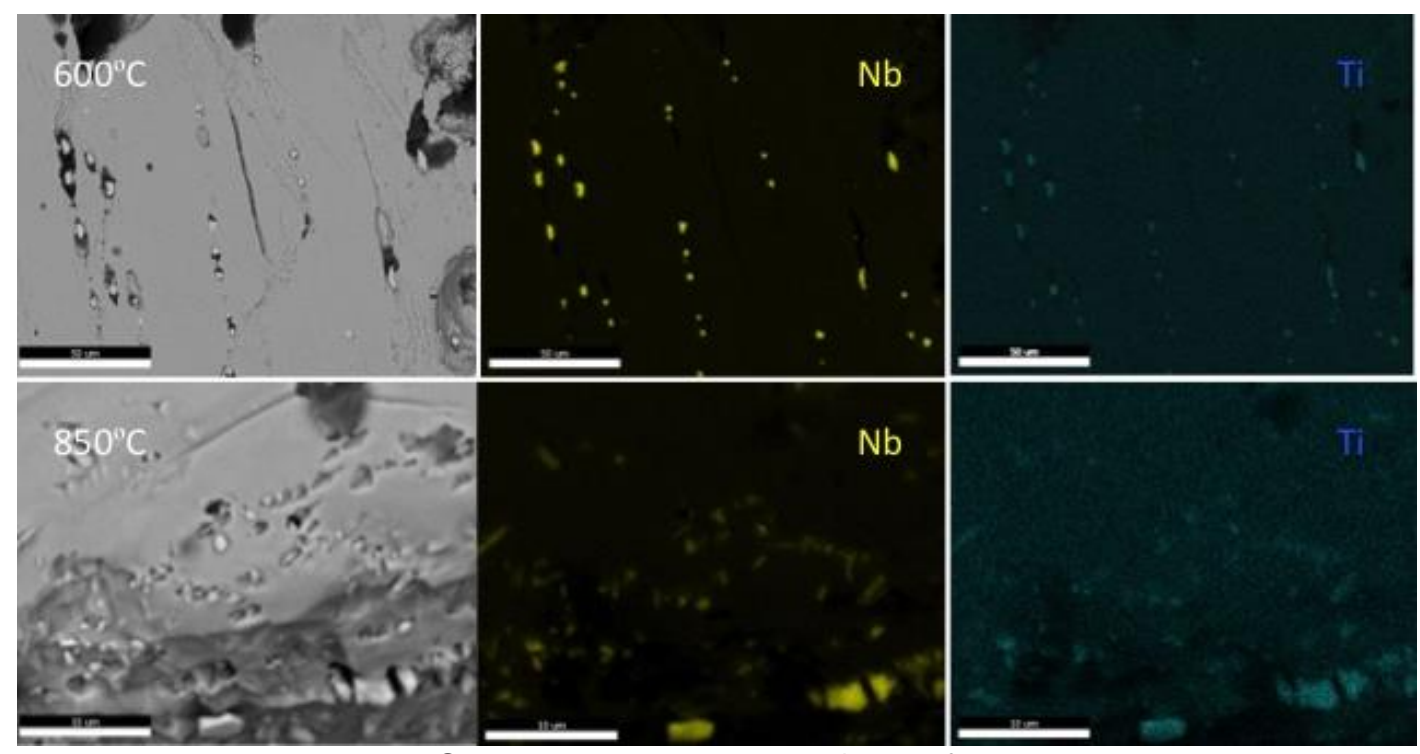

Figura 5 - Mapeamento por EDS da Inconel 718 nas regiões de fratura, destacando os sinais de $\mathrm{Nb}$ e Ti. Acredita-se tratar de carbetos $(\mathrm{Nb}, \mathrm{Ti}) \mathrm{C}$ atuando no mecanismo de fragilização.

\section{CONCLUSÃO}

Foi identificada uma clara transição contínua no modo de fratura da Inconel 718 com a temperatura, que se iniciou nas bordas do corpo de prova em direção ao seu interior. 
Quanto maior a taxa de deformação, ou mais rápida, maior foi a temperatura de início identificada para o OAIC, como esperado por ser um fenômeno termicamente ativado dependente de tempo e temperatura.

Em todas as taxas avaliadas, o fenômeno teve fim aproximado na mesma temperatura, de $900^{\circ} \mathrm{C}$, indicando que a formação da camada de óxido estável acontece independente do tempo.

Nióbio foi encontrado ao redor de trincas secundárias em toda a região de fratura, incorporado ao titânio, indicando tratar-se de carbetos MC do tipo (Nb,Ti)C.

Como os ensaios mecânicos foram realizados em vácuo e, portanto, com a disponibilidade de oxigênio reduzida, os resultados sugerem que essa disponibilidade não é crucial para o aparecimento do fenômeno de fragilização da liga.

\section{Agradecimentos}

À Capes (2015) e Faperj (2016).

\section{REFERÊNCIAS}

1 Castro L De, Valle M. Efeitos da Solubilização e do Envelhecimento na Microestrutura e nas Propriedades Mecânicas da Superliga Inconel 718. 2010.

2 Rezende MC, Araújo LS, Gabriel SB, Dille J, de Almeida LH. Oxidation assisted intergranular cracking under loading at dynamic strain aging temperatures in Inconel 718 superalloy. J Alloys Compd. 2015. Acesso em 20/04/2015; 643: S256-S259. Disponível em: http://dx.doi.org/10.1016/j.jallcom.2014.12.279

3 Gao M, Wei RP. Grain Boundary Niobium Carbides In Inconel 718. Scr Mater. 1997;6462(97):1843-9

4 Sundararaman M, Mukhopadhyay P, Banerjee S. Carbide precipitation in Nickel Base Superalloys 718 and 625 and their effect on mechanical properties. Superalloys 718, 625, 706 Var Deriv. 1997;367-78.

5 Araújo LS. Processamento e caracterização de superliga de níquel 718 para aplicações nucleares. Vol. 1. 2012.

6 Woodford D a. Gas phase embrittlement and time dependent cracking of nickel based superalloys. Energy Mater Mater Sci Eng Energy Syst. 2006;1(1):59-79.

7 Gao M, Dwyer DJ, Wei RP. Chemical and Microstructural Aspects of Creep Crack Growth in Inconel 718 Alloy. Superalloys 718, 625, 706 Var Deriv. 1994;581-92.

8 Valerio P, Gao M, Wei RP. Environmental Enhancement of Creep Crack Growth in Inconel 718 by Oxygen and Water Vapor. Scr Metall Mater. 1994;30(10):1269-74.

9 Delaunay F, Berthier C, Lenglet M, Lameille J-M. SEM-EDS and XPS Studies of the High Temperature Oxidation Behaviour of Inconel 718. Microchim Acta [Internet]. 2000;132(2-4):337-43. 